\author{
J.K. Chen \\ W.Y. Duan
}

http://dx.doi.org/10.21278/brod71407

ISSN 0007-215X

eISSN $1845-5859$

\title{
A NOVEL METHOD FOR THE M-TERMS OF SHIP WITH FORWARD SPEED
}

UDC 629.5.017.2:629.543

Original scientific paper

\begin{abstract}
Summary
One of the major difficulties in linear wave-induced ship motion problem with forward speed is how to solve the $m$-terms accurately. This paper proposes a novel numerical method (Taylor Expansion Boundary Element Method, TEBEM) to compute the $m$-terms for arbitrary floating bodies. This method treats the $m$-terms as the Dirichlet type, uses the first-order derivatives terms on the right-handed side of boundary value problem, which is solved by TEBEM method. Numerical studies are performed for the hemisphere, mounted cylinder, and modified KVLCC2 ship models. Compared to the analytical solutions and other numerical results, a good agreement can be obtained by the TEBEM method.
\end{abstract}

Key words: m-terms; Taylor expansion boundary element method; second-order derivative; forward speed; sharp corner

\section{Introduction}

When the linear wave-induced ship motion problem with forward speed is considered, the interaction between the local steady flow and unsteady wave field should be considered in both the body surface and free surface boundary condition for the unsteady boundary value problem (BVP). The $m$-terms involved in the body surface condition is the second order derivatives of the velocity potential substantially. It is difficulty to calculate accurately for any ship body. With the increase in computer power, many researchers choose the CFD method to study the ship motion and added resistance, such as Lee et al [1], and avoid to calculate the $m$-terms involved in the BVP of ships with forward speed.

Faltinsen (1974) [2] considered the influence of the $m$-terms in the calculation of the added mass and damping coefficient of the ships with forward speed. Many references have revealed the importance of $m$-terms, such as Inglis and Price (1981) [3], Iwashita and Bertram (1997) [4], Chen and Malenica (1998) [5], Duan and Price (2002) [6] etc. Iwashita and Bertram (1997) [4] pointed out the influence of $m$-terms on the wave pressure near the bow region. Chen and Malenica (1998) [5] found $m$-terms play an important role in the solutions of the added mass and damping coefficient by the numerical method inspired from Wu (1991) 
[7]. Duan and Price (2002) [5] even found that the local steady flow make a significant contribution to the slender body around the bow and stern.

The $m$-terms are usually neglected when taking the steady potential as the incoming uniform stream for the slender ship under the assumption of high speed. However, the impact cannot be ignored for the blunt ship with low forward speed. There are several attempts to deal with $m$-terms, which can be divided into the indirect method and direct method. For the indirect method, Bai (2000) [8], Teng et al. (2002) [9] and Kim et al. (2011) [10] all applied a modified Stokes formula to rewrite the effect of the second-order derivatives in $m$-terms in terms of the first-order derivatives and the corresponding waterline integral which involves additionally the first-order derivatives of the Green function. Shao and Faltinsen (2012) [11] calculated the added resistance of the ships under the body-fixed coordinate system without the second-order derivatives of the steady velocity potential on the body surface condition.

For the direct method, Zhao and Faltinsen (1989) [12] calculated the second-order derivatives at these points, which are offset in the fluid domain, because the singularity of Rankine source is weaken away from the boundary surface. The $m$-terms on the mean wetted body surface can be obtained by extrapolation. Raven (1996) [13] applied the desingularized panel method to calculate the wave-making resistance, whose kernel is making the singularities offset outside of the fluid domain to avoid the difficulty of dealing with the singularity of Green function, then the derivatives can be computed by differentiating the Green function. Nonetheless, the offset distance should be set carefully. Wu (1991) [7] handled the $m$-terms as Dirichlet type on the basis that the first-order derivatives could be accurately computed. Bingham and Maniar (1996) [14] represented the geometry and velocity potential by sixth-order B-splines method, which is solved by the higher-order boundary element method (HOBEM), then the first-order derivatives and $m$-terms could be evaluated by differentiating the shape functions. These methods could only offer satisfactory accuracy for the wave-body-current interactions problem without sharp corners.

How to calculate the $m$-terms for arbitrary floating bodies is the purpose of this paper. A novel BEM method is proposed to solve the BVP, named the Taylor Expansion Boundary Element Method (TEBEM), which can calculate the potential and velocity accurately for non-smoothed boundary, such as the ship structures. Then solve the second-order derivatives as solutions of a problem of the Dirichlet type. The mathematical formulations of $\mathrm{m}$-terms are briefly reviewed in section 2 . The scheme for calculating $m$-terms by the TEBEM is discussed in section 3. In section 4, the numerical issues of the hemisphere, the mounted cylinder, modified KVLCC2 ship models will show the superiority of the TEBEM.

\section{Mathematica formulas for m-terms}

A moving system of coordinates $o-x y z$ in steady translation with forward speed $U$ is introduced. The xoy plane lies in the undisturbed free surface with the origin $o$ at the projective point of the gravity center on this surface, the positive $o x$ axis is in the direction of forward speed and the positive $o z$ axis is vertically upwards. The fluid is assumed incompressible, inviscid, and irrotational, and the steady velocity potential $\bar{\phi}$ can be described as follows:

1) Based on the steady Double-Body (DB) assumption, the steady potential $\bar{\varphi}$ satisfies 
the Laplace equation:

$$
\frac{\partial^{2} \bar{\phi}}{\partial x^{2}}+\frac{\partial^{2} \bar{\phi}}{\partial y^{2}}+\frac{\partial^{2} \bar{\phi}}{\partial z^{2}}=0
$$

in the whole fluid domain.

2) The kinematics condition on the mean wetted body surface $S_{H}$ is governed by the relation

$$
\frac{\partial \bar{\phi}}{\partial n}=\vec{U} \cdot \vec{n}
$$

Where $\vec{n}=\left(n_{1}, n_{2}, n_{3}\right)$ is the normal vector, which points to the outside of the fluid domain.

3) On the undisturbed free surface, $z=0$

$$
\frac{\partial \bar{\phi}}{\partial z}=0
$$

4) In water of finite depth, the condition is

$$
\frac{\partial \bar{\phi}}{\partial z}=0
$$

for $z \rightarrow h$

Consequently, the steady potential $\bar{\phi}$ can be solved based on the above boundary value problem. For the unsteady problem of ships with forward speed, it is necessary to consider the impact of the steady potential for the body surface condition in the unsteady boundary value problem. The displacement of the ship is as following:

$$
\begin{aligned}
& d=\xi+\theta \times r \\
& \xi=\left(\eta_{1}, \eta_{2}, \eta_{3}\right), \theta=\left(\eta_{4}, \eta_{5}, \eta_{6}\right)
\end{aligned}
$$

Where $\xi$ and $\theta$ mean the translational and the rotational displacement respectively, $r=(x, y, z)$ is the position vector. In other words, it can be represented as following:

$$
\begin{aligned}
& d=\sum_{k=1}^{6} \eta_{k} u_{k}, \theta=\sum_{k=4}^{6} \eta_{k} u_{k-3} \\
& u_{1}=(1,0,0), u_{4}=i \times r=(0,-z, y), \\
& u_{2}=(0,1,0), u_{5}=j \times r=(z, 0,-x), \\
& u_{3}=(0,0,1), u_{6}=k \times r=(-y, x, 0)
\end{aligned}
$$

For unsteady boundary value problem, the condition on the body surface is:

$$
\vec{w} \cdot \vec{n}+\sum_{k=1}^{6} \frac{\partial \tilde{\phi}_{k}}{\partial n}=\sum_{k=1}^{6} \dot{\eta}_{k} n_{k}\left(\text { on } s_{H}(t)\right)
$$

Where $\tilde{\phi}$ and $\eta$ are both first-order infinitesimal quantities, but the steady velocity $\vec{w}=\nabla(\bar{\phi}-U)$ is not the infinitesimal. Taylor expansion is applied and the second-order and high-order terms are all neglected.

$$
\begin{aligned}
& \left.w\right|_{s_{H}(t)}=\left.w\right|_{s_{H}}+\left.(d \cdot \nabla) w\right|_{s_{H}} \\
& \left.n\right|_{s_{H}(t)}=\left.n\right|_{s_{H}}+\left.(\theta \times n)\right|_{s_{H}}
\end{aligned}
$$


Then $\vec{w} \cdot \vec{n}$ can be expressed on the static water plane as following:

$$
\left.\vec{w} \cdot \vec{n}\right|_{s_{H}(t)}=\left.\{\vec{w} \cdot \vec{n}-(\theta \times \vec{w}) \cdot \vec{n}+[(d \cdot \nabla) \vec{w}] \cdot \vec{n}\}\right|_{s_{H}}=-U \sum_{k=1}^{6} m_{k} \eta_{k}
$$

Where $m_{k}=\left\{\begin{array}{l}-\left[\left(u_{k} \cdot \nabla\right) \vec{w}\right] \cdot \vec{n} / U(k=1,2,3) \\ \left\{-\left[\left(u_{k} \cdot \nabla\right) \vec{w}\right] \cdot \vec{n}-\left(u_{k-3} \times w\right) \cdot n\right\} / U(k=4,5,6)\end{array}\right.$.

Finally, the body surface boundary condition for unsteady boundary value problem can be written as:

$$
\frac{\partial \tilde{\phi}_{k}}{\partial n}=\dot{\eta}_{k} n_{k}+U \eta_{k} m_{k}\left(k=1 \sim 6, \text { on } s_{H}\right)
$$

The well-known $m$-terms in details are given as

$$
\begin{aligned}
& m_{1}=-\left(\bar{\phi}_{x x} n_{x}+\bar{\phi}_{x y} n_{y}+\bar{\phi}_{x z} n_{z}\right) / U \\
& m_{2}=-\left(\bar{\phi}_{x y} n_{x}+\bar{\phi}_{y y} n_{y}+\bar{\phi}_{y z} n_{z}\right) / U \\
& m_{3}=-\left(\bar{\phi}_{x x} n_{x}+\bar{\phi}_{x y} n_{y}+\bar{\phi}_{x z} n_{z}\right) / U \\
& m_{4}=y m_{3}-z m_{2}-\left(\bar{\phi}_{z} n_{y}-\bar{\phi}_{y} n_{z}\right) / U \\
& m_{5}=z m_{1}-x m_{3}-\left(\left(\bar{\phi}_{x}-U\right) n_{z}-\bar{\phi}_{z} n_{x}\right) / U \\
& m_{6}=x m_{2}-y m_{1}-\left(\bar{\phi}_{y} n_{x}-\left(\bar{\phi}_{x}-U\right) n_{y}\right) / U
\end{aligned}
$$

While based on Neumann-Kelvin (NK) assumption, the m-terms can be obtained as follows:

$$
\begin{aligned}
& m_{1}=m_{2}=m_{3}=m_{4}=0 \\
& m_{5}=U n_{3}, m_{6}=-U n_{2}
\end{aligned}
$$

\section{The scheme for calculating m-terms by the TEBEM}

Here, the steady potential and its first-order derivatives have been solved accurately for arbitrary floating body by the TEBEM, which can be obtained from reference [15]. Then the $m$-terms can be solved based on the accurate first-order derivatives. The scheme is shown as following.

Firstly, it introduces a new potential function $\psi=\bar{\phi}_{, x}$. Green's third formula is applied to $\psi$, when the field point locates in the external domain of the water, and gets

$$
\iint_{S} \psi(q) \cdot G_{, n_{q}}(p, q) d s_{q}=\iint_{S} G(p, q) \cdot \psi_{, n}(q) d s_{q}
$$

Where $S=S_{H}+S_{F}$, the subscript ',' means the differentiation, such as ',n ' is the normal derivative. $G$ is Green function; $1 / r$ is Rankine source, in which $r$ represents the distance between the source point $q(\xi, \eta, \zeta)$ and the field point $p(x, y, z)$.

The whole boundary is divided into $N$ small flat surface elements. Define $(\bar{\xi}, \bar{\eta}, \bar{\zeta})$ as the 
local coordinates of the source point $q$ in element Q (Referring to the Fig. 1, where the direction of $\bar{\zeta}$ is the same with $\left.\vec{n}_{q}\right)$. Define $(\bar{x}, \bar{y}, \bar{z})$ as the local coordinates of the field point $p$ in element $\mathrm{P}$, where the direction of $\bar{z}$ is the same with $\vec{n}$. Meanwhile, the Taylor expansion is applied for the dipole strength in each small element, and only the first-order derivatives are reserved.

$$
\psi(q)=\psi\left(q_{0}\right)+\psi_{, \bar{\xi}}\left(q_{0}\right) \bar{\xi}+\psi_{, \bar{\eta}}\left(q_{0}\right) \bar{\eta}
$$

Fig.1. The sketch of the local coordinate system

Substitute Eq. (14) into the dipole strength in Eq. (13). The Cauchy Principal Value (CPV) of the BIE is $2 \pi$, and the new BIE can be obtained for the potential function $\psi$ as follows

$$
\begin{aligned}
& -\sum_{j=1}^{N} \psi_{, \bar{\zeta}}\left(q_{0}\right) \cdot \iint_{\Delta S_{j}} G(p, q) d s+\sum_{j=1}^{N} \psi_{, \bar{\xi}}\left(q_{0}\right) \cdot \iint_{\Delta S_{j}} \bar{\xi} G_{, \bar{\zeta}}(p, q) d s \\
& +\sum_{j=1}^{N} \psi_{, \bar{\eta}}\left(q_{0}\right) \cdot \iint_{\Delta S_{j}} \bar{\eta} G_{, \bar{\zeta}}(p, q) d s=-\sum_{j=1, j \neq i}^{N} \psi\left(q_{0}\right) \cdot \iint_{\Delta S_{j}} G_{, \bar{\zeta}}(p, q) d s-2 \pi \psi(p)
\end{aligned}
$$

For this Dirichlet type BVP, the other $2 N$ equations are needed to form the closed equations. Hence, the tangential first-order derivatives of the field point $p$ at $\bar{x}$ and $\bar{y}$ direction for Eq. (7) are adopted and the Taylor expansion procedure as Eq. (14) is applied to the dipole strength. The supplementary equations are

$$
\begin{aligned}
& 2 \pi \psi_{, \bar{x}}(p)-\sum_{j=1}^{N} \psi_{, \bar{\zeta}}\left(q_{0}\right) \cdot \frac{\partial}{\partial \bar{x}} \iint_{\Delta S_{j}} G(p, q) d s+\sum_{j=1, j \neq i}^{N} \psi_{, \bar{\xi}}\left(q_{0}\right) \cdot \frac{\partial}{\partial \bar{x}} \iint_{\Delta S_{j}} \bar{\xi} G_{, \bar{\zeta}}(p, q) d s \\
& +\sum_{j=1}^{N} \psi_{, \bar{\eta}}\left(q_{0}\right) \cdot \frac{\partial}{\partial \bar{x}} \iint_{\Delta S_{j}} \bar{\eta} G_{, \bar{\zeta}}(p, q) d s=-\sum_{j=1}^{N} \psi\left(q_{0}\right) \cdot \frac{\partial}{\partial \bar{x}} \iint_{\Delta S_{j}} G_{\bar{\zeta}}(p, q) d s \\
& 2 \pi \psi_{, \bar{y}}(p)-\sum_{j=1}^{N} \psi_{, \bar{\zeta}}\left(q_{0}\right) \cdot \frac{\partial}{\partial \bar{y}} \iint_{\Delta S_{j}} G(p, q) d s+\sum_{j=1}^{N} \psi_{, \bar{\xi}}\left(q_{0}\right) \cdot \frac{\partial}{\partial \bar{y}} \iint_{\Delta S_{j}} \bar{\xi} G_{, \bar{\zeta}}(p, q) d s \\
& +\sum_{j=1, j \neq i}^{N} \psi_{, \bar{\eta}}\left(q_{0}\right) \cdot \frac{\partial}{\partial \bar{y}} \iint_{\Delta S_{j}} \bar{\eta} G_{, \bar{\zeta}}(p, q) d s=-\sum_{j=1}^{N} \psi\left(q_{0}\right) \cdot \frac{\partial}{\partial \bar{y}} \iint_{\Delta S_{j}} G_{, \bar{\zeta}}(p, q) d s
\end{aligned}
$$

Where all the influence coefficients can be evaluated analytically on each element. The procedure to compute the influence coefficient is shown in the reference [15]. The gradients of $\psi$ can be obtained by solving the combining equation set (15), (16) and (17). Then we also 
assume that $\psi=\bar{\phi}_{, y}$ and $\psi=\bar{\phi}_{, z}$. Finally, the whole terms of double gradients and $m$-terms can be solved accurately.

\section{Numerical results and discussion}

\subsection{Hemisphere model}

To examine the validity of TEBEM method, the case of a hemisphere of radius $R=1.0 \mathrm{~m}$ floating in infinite depth is considered. The analytical solution of the double-body flow velocity potential $\bar{\phi}$ for hemisphere can be expressed as:

$$
\bar{\phi}(x, y, z)=-\frac{U R^{3}}{2} \frac{x-x_{0}}{\left(\left(x-x_{0}\right)^{2}+\left(y-y_{0}\right)^{2}+\left(z-z_{0}\right)^{2}\right)^{3 / 2}}
$$

Where $\left(x_{0}, y_{0}, z_{0}\right)$ means the origin of the hemisphere on the plane xoy.

Source Method (SM) and TEBEM method are applied to calculate the $m$-terms respectively. In view of the symmetry current, Green function is $1 / r+1 / r^{\prime}$ here. There are total 300 quadrilateral panels on the hemisphere. Fig. 2 and 3 show the $m_{1}$ and $m_{3}$ at the centre of mesh in the plane of $\mathrm{z}=-0.0775$ near the free surface calculated by two methods. Compared with the analytical solutions, it is found that the results of the TEBEM and SM have a good agreement with the analytical solutions.

We also apply Green function $1 / r$ to solve this issue by two methods. Hence, it is necessary to arrange the singularity points on the free surface. The radius of free surface zone is $R_{f}=10 R$, where the influence of the truncated free surface zone can be ignored due to the influence of local flow decrease rapidly with the distance from hemisphere. There are 640 quadrilateral discretization panels on the free surface. Fig. 4 and 5 show the $m_{1}$ and $m_{3}$ at the same centroids for the model of hemisphere with free surface. Compared to the results of SM, a good agreement can be obtained between numerical results of TEBEM and analytical solution. For the $m_{3}$, it can be easily found that the discrepancy of SM is induced for the first-order derivative $\bar{\phi}_{z,}$ in Eq. (12). Compared with TEBEM, the $\bar{\phi}_{z z}$ calculated by SM is not accurate enough for the sharp corners near the interaction between free surface and body surface. 


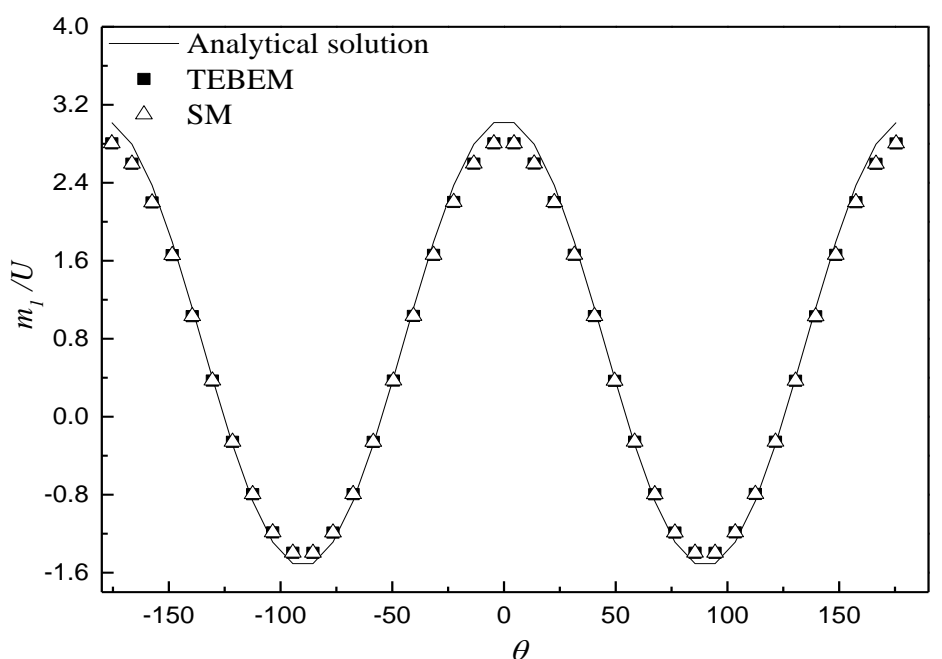

Fig.2. The values of $m_{1}$ at the center of mesh in the plane of $\mathrm{z}=-0.0755$ by SM and TEBEM, G $=1 / r+1 / r^{\prime}$

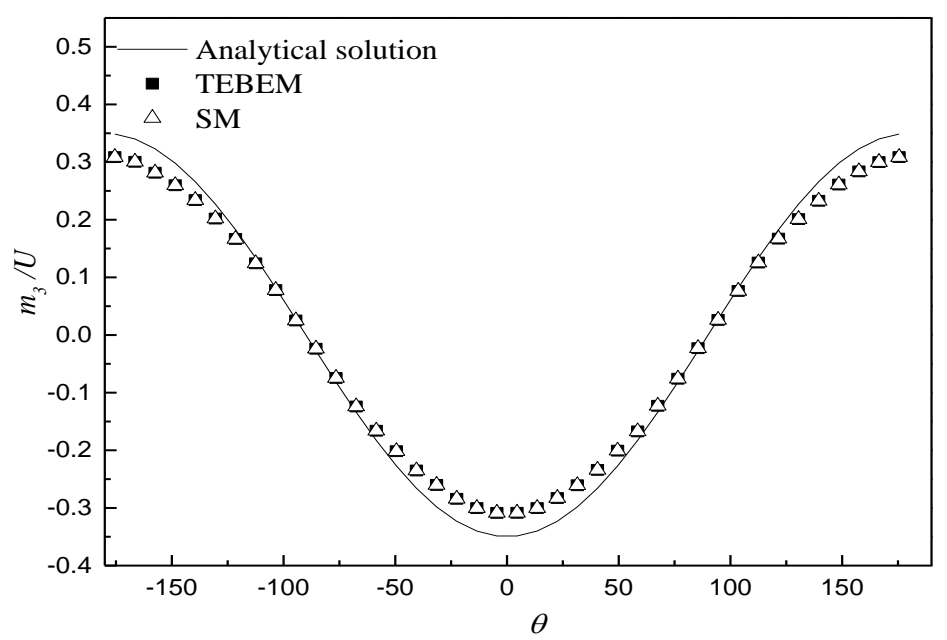

Fig.3. The values of $m_{3}$ at the center of mesh in the plane of $\mathrm{z}=-0.0755$ by $\mathrm{SM}$ and TEBEM, $\mathrm{G}=1 / r+1 / r^{\prime}$

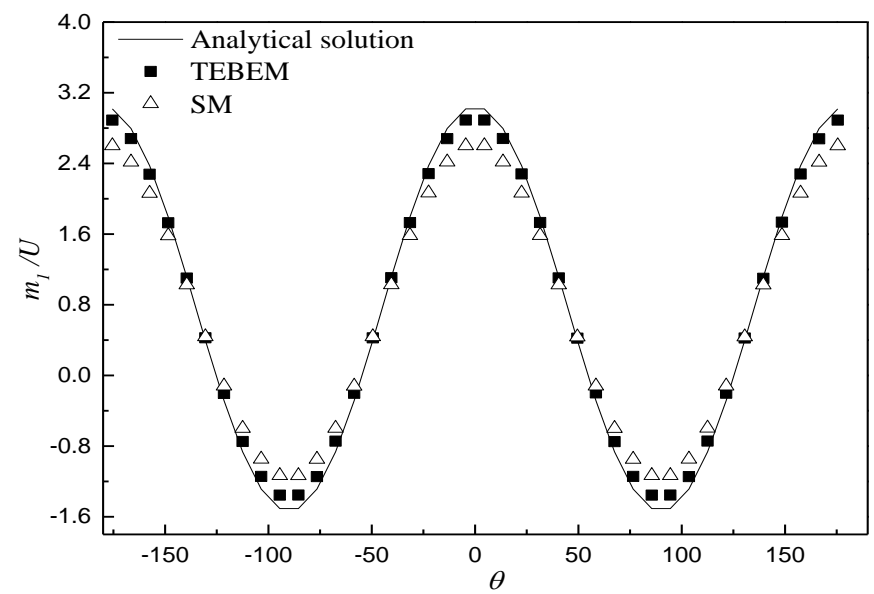

Fig.4. The values of $m_{1}$ at the center of mesh in the plane of $\mathrm{z}=-0.0755$ by $\mathrm{SM}$ and TEBEM, $\mathrm{G}=1 / r$ 


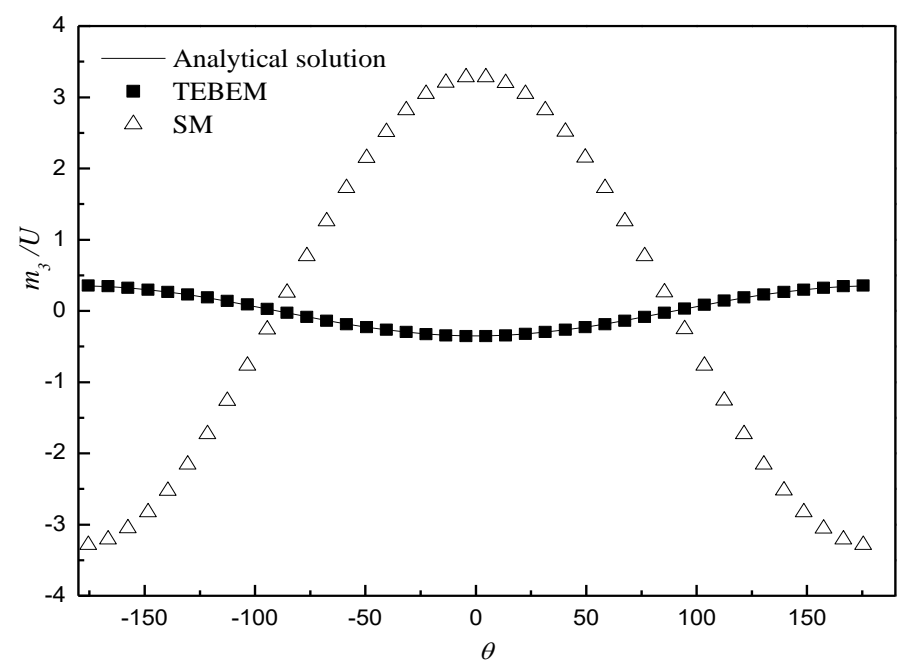

Fig.5. The values of $m_{3}$ at the center of mesh in the plane of $\mathrm{z}=-0.0755$

by $\mathrm{SM}$ and TEBEM, $\mathrm{G}=1 / r$

In order to verify the convergence rate of the TEBEM. The $\mathrm{L}_{2}$ errors of the $m_{3}$ terms on the hemisphere are shown in Fig. 6. Here, the $\mathrm{L}_{2}$ error for a function $f$ is defined as:

$$
L_{2} \text { error }=\sqrt{\sum_{j=1}^{N}\left(f_{\text {ana }}^{j}-f_{\text {num }}^{j}\right)^{2} / \sum_{j=1}^{N}\left(f_{\text {ana }}^{j}\right)^{2}}
$$

Where $f_{\text {ana }}^{j}$ and $f_{\text {num }}^{j}$ are the analytical and numerical solutions at the $j$ th element centre respectively. The $\mathrm{L}_{2}$ error are plotted versus the function $\sqrt{\Delta s} / R, \Delta s$ means the average area of the panels on the hemisphere. The linear fitting of error in Fig. 6 are shown below

$$
\left[L_{2} \text { error }\right]_{m 3}=-1.75 e^{-4}+0.46(\sqrt{\Delta s} / R)
$$

The present TEBEM has only $\mathrm{j}=1$ convergence rate and the slope is 0.46 for $m_{3}$ term. We could find the TEBEM method could retain the contribution of first and second order derivatives of the dipole strength, which could improve the accuracy and convergence rate of the velocity potential, the corresponding information can be referred to Duan (2015) [15]. However, the $m$-terms involve the second-order derivatives of the velocity potential. Compared with the velocity potential, the accuracy of second-order derivatives would decrease, as expected. 


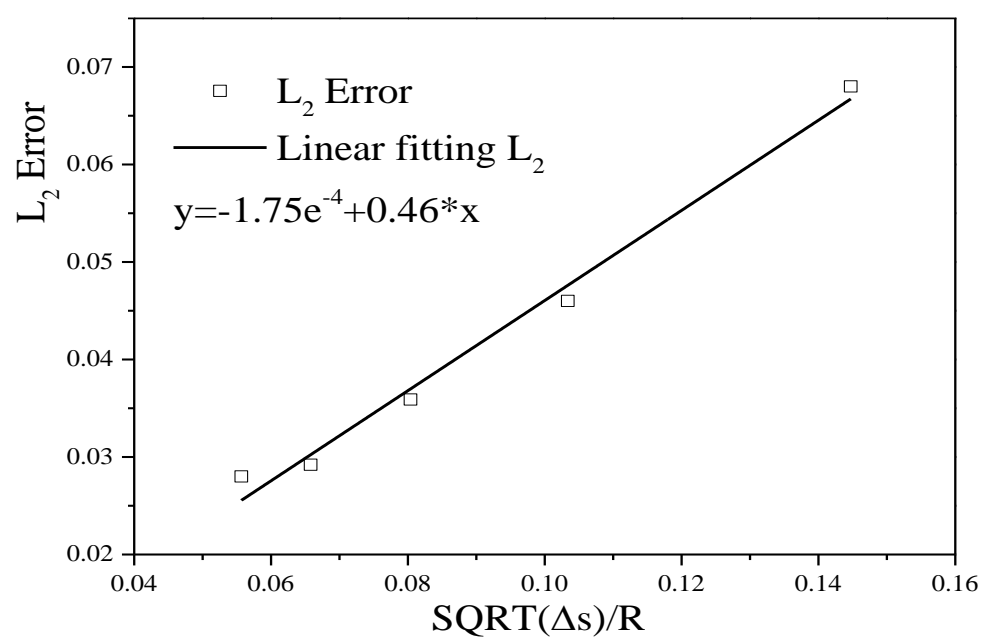

Fig.6. The $\mathrm{L}_{2}$ errors of the $\mathrm{m}_{3}$ term on the hemisphere surface by TEBEM, $\mathrm{G}=1 / \mathrm{r}$

\subsection{Mounted cylinder model}

Considering the influence of the current, diffraction problem of a bottom mounted cylinder has been studied by many researchers. Here, the same numerical example is solved by the TEBEM. The water depth, radius and draft of the cylinder are denoted by $H, R$ and $D$, where $H=R=D=1.0$. The radius of free surface is set as $R_{f}=10 R$. The detailed information is illustrated in Fig. 7. Here, there are 500 and 2000 quadrilateral discretization panels on the body and free surface, respectively.

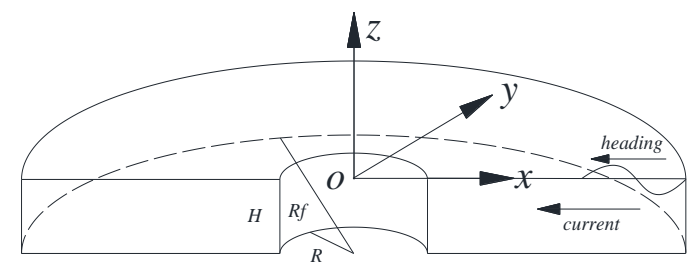

Fig.7. Sketch of the computational parameters for the mounted cylinder

The analytical solution of the double-body flow velocity potential $\bar{\phi}$ for the mounted cylinder can be expressed as,

$$
\bar{\phi}(x, y, z)=-\frac{U\left(x-x_{0}\right) R^{2}}{\left(x-x_{0}\right)^{2}+\left(y-y_{0}\right)^{2}}
$$

Where $\left(x_{0}, y_{0}\right)$ means the position of the cylinder axis in the horizontal plane. 


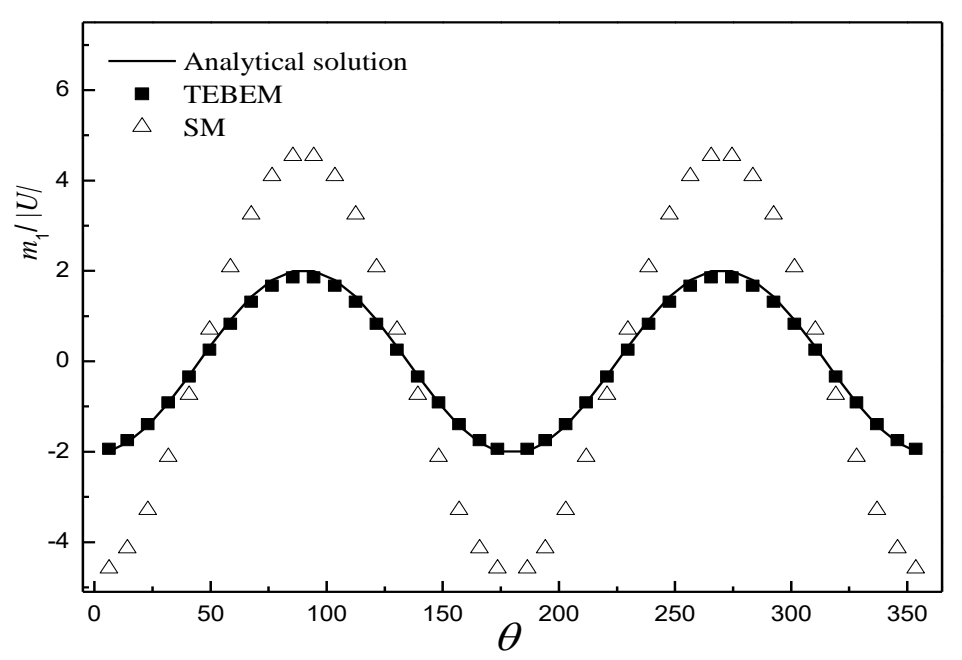

Fig.8. $m_{1}$ at the center of mesh in the plane of $\mathrm{z}=-0.05$ by TEBEM and $\mathrm{SM}, \mathrm{G}=1 / \mathrm{r}$

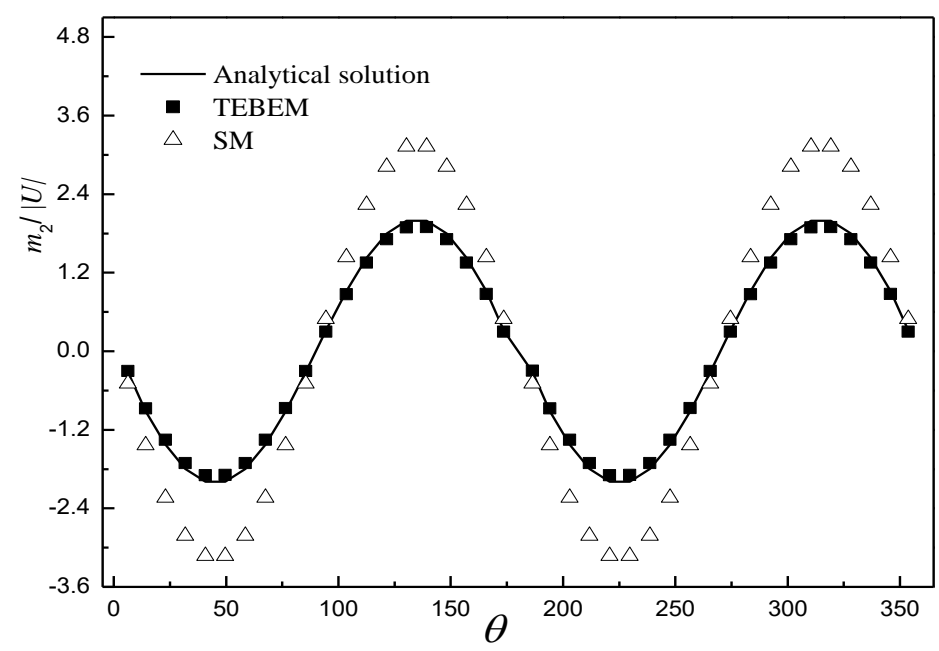

Fig.9. $m_{2}$ at the center of mesh in the plane of $\mathrm{z}=-0.05$ by TEBEM and $\mathrm{SM}, \mathrm{G}=1 / \mathrm{r}$

Fig. 8 and 9 show the $m_{1}$ and $m_{2}$ at the centre of mesh in the plane of $\mathrm{z}=-0.05$ calculated by the TEBEM and Wu formula by SM method (1991) [7]. $\theta$ means the angle of the location on the mounted cylinder with respect to the $x$-axis. It is found that the results of the TEBEM have a good agreement with the analytical solutions and are better than those of the SM method. Because there are sharp corners between the body- and free-surface, TEBEM can compute accurately the induced velocity for the fluid boundary with sharp corners. Consequently, the accuracy of the $m$-terms solved by TEBEM is better.

For the hemisphere and mounted cylinder cases, we could find that the sharp corners would form near the body- and free-surface domain without the applying of the imaging Green function. The induced velocity and second-order derivatives of velocity potential are singularity on the sharp corner curve. Hence, they can't be solved on the sharp corner curve through the Taylor expansion and perturbation method, which also means that $m$-terms on the 
sharp corner curve cannot be calculated. In TEBEM method, the collect singular points are all arranged at the centre of the elements, would not locate on the sharp corner curve, and the boundary value problem obtained by Taylor expansion and perturbation method can be solved in theory. Compared with SM method, the rate of change of the dipole strength is considered in TEBEM, and details on how TEBEM improve the accuracy of the induced velocity near the sharp corner have been discussed in Duan (2020) [16].

\subsection{KVLCC2 ship model}

The influence of the m-terms for ship motion is discussed in this section. Here the modified KVLCC2 ship is taken as an example. Yasukawa (2019) [17] developed the seakeeping and manoeuvring research in the physical tank. The main dimensions of the modified KVLCC2 ship are shown in Table 1 as following:

Table 1 The main dimensions of modified KVLCC2 ship

\begin{tabular}{l|c|c}
\hline & Full scale & model \\
\hline Ship Length, m & 178.0 & 2.909 \\
\hline Breath, m & 32.26 & 0.527 \\
\hline Draught, m & 11.57 & 0.189 \\
\hline Radius of inertia, m & $10.0,44.5,44.5$ & $0.163,0.727,0.727$ \\
\hline $\begin{array}{l}\text { Center of gravity, m } \\
\text { (Based on the water surface) }\end{array}$ & $5.33,0.0,-4.51$ & $0.087,0.0,-0.074$ \\
\hline Block coefficient & 0.84 & 0.84 \\
\hline Metacentric height, $\mathrm{m}$ & 6.61 & 0.108 \\
\hline Roll natural period, $\mathrm{s}$ & 9.0 & 1.15 \\
\hline
\end{tabular}

We developed the in-house code to predict the ship motion, which the principle could refer to Duan 2020 [18]. Fig. 10 and 11 show the comparison of ship heave motion amplitudes between DB and NK scheme with different forward speed in the heading waves by TEBEM method. Here, heave and pitch motion are non-dimensional by $h_{a}$ and $K h_{a} . h_{a}$ means the amplitude of the incident wave. $\mathrm{K}$ and $\lambda$ are the wave number and length of the incident wave. Compared with the experimental solutions, agreement of numerical results of DB scheme is better than NK scheme. It should be pointed out that the difference between DB and NK scheme for ship motion involves two aspects, one is the $m$-terms in the body surface condition, the other is the convection term in the free surface condition.

Compared with the pitch motion, the heave motion show larger discrepancy between the DB and NK scheme during the resonant frequency domain especially. Whereas in the high frequency domain, the ship motion is smaller naturally, and the diffraction impaction is weaker in the low frequency domain, hence the difference of ship motion between DB and 
NK scheme can be neglected.

Finally, we also made the comparison of the ship motion for different forward speeds. It can be found that the greater the forward speed, the larger the difference between DB and NK scheme, and the $m$-terms play an important role in the ship motions especially for the high forward speed condition.

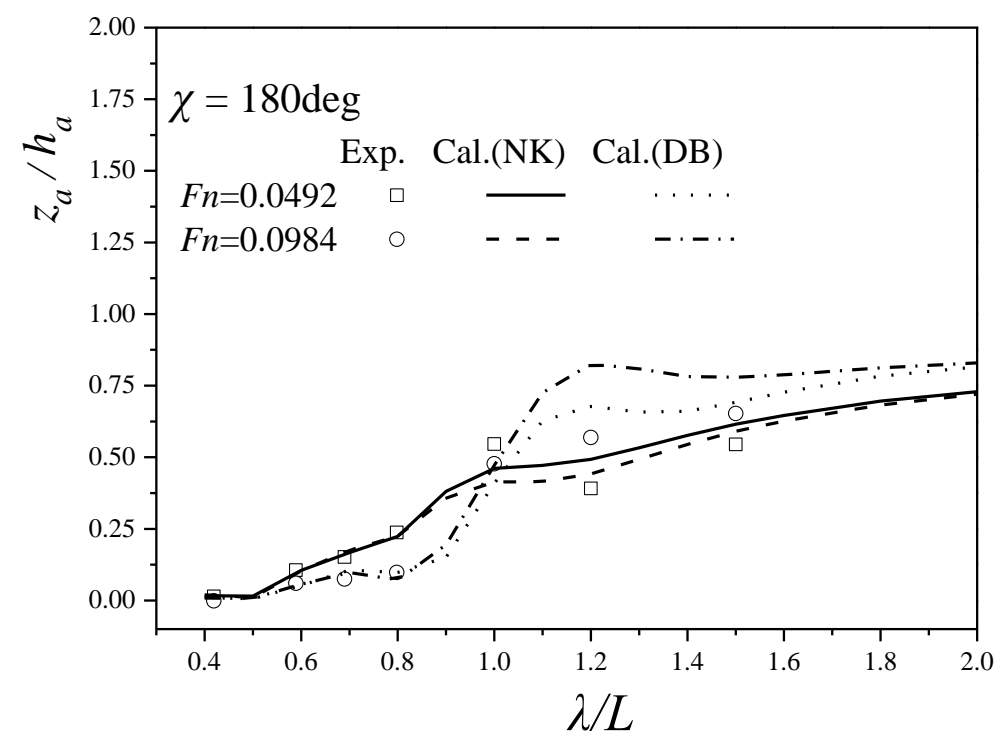

Fig.10. Comparison of ship heave motion amplitudes between DB and NK scheme by TEBEM method for different forward speed.

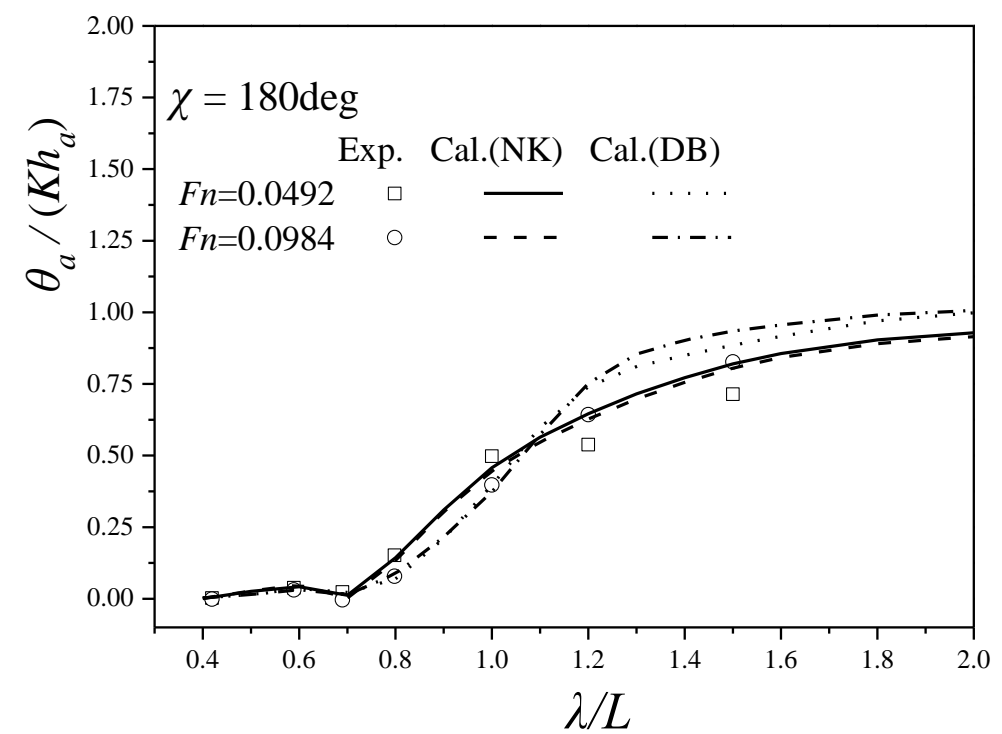

Fig.11. Comparison of ship pitch motion amplitudes between DB and NK scheme by TEBEM method for different forward speed.

\section{Conclusions}

This paper introduces a new method, named the TEBEM method, to solve the $m$-terms precisely, which involves in the wave-induced motion problem of the floating body with 
forward speed. It is validated by the numerical results of the hemisphere, the mounted cylinder, and the modified KVLCC2 ship models. The main conclusions can be obtained are as follows:

(1) Compared with the analytical solutions of the hemisphere and the mounted cylinder, it is demonstrated that the proposed method could compute precisely $m$-terms for any boundaries with sharp corners.

(2) For DB assumption, a good agreement can be obtained for the unsteady ship motion. Large difference is shown in the resonant frequency domain notably. The greater the forward speed, the more important role $m$-terms play in ship motions.

\section{Acknowledgement}

The authors acknowledge financial support from the National Natural Science Foundation of China (Grant No. 51709064, 51679043, 51779050) and the Numerical Tank Project sponsored by the Ministry of Industry and Information Technology (MIIT) of P.R. China.

\section{REFERENCES}

[1] Lee Y.G., Kim C., Park J.H., Kim H., Lee I., and Jin B., Numerical simulations of added resistance in regular head waves on a container ship. Brodogradnja, 70(2), 61-85, 2019. https://doi.org/10.21278/brod70204

[2] Faltinsen O.M., A numerical investigation of the Ogilvie-Tuck formulas for added-mass and damping coefficients, Journal of ship research, 18, 73-84, 1974.

[3] Inglis R.B. and Price W.G., The influence of speed dependent boundary condition in three-dimensional ship motion problems, International shipbuilding progress, 28(318), 22-29, 1981. https://doi.org/10.3233/ISP-1981-2831801

[4] Iwashita H. and Bertram V., Numerical study on the influence of the steady flow in seakeeping, 12th international workshop on water wave and floating bodies, France, 1997.

[5] Chen X.B. and Malenica S., Interaction effects of local steady flow on wave diffraction-radiation at low forward speed, International journal of offshore and polar engineering, 8(2), 102-109, 1998.

[6] Duan W.Y. and Price W.G., A numerical method to solve the mj terms of a submerged body with forward speed, International journal for numerical methods in engineering, 40, 655-667, 2002. https://doi.org/10.1002/fld.367

[7] $\mathrm{Wu}$ G.X., A numerical scheme for calculating the m-terms in wave-current-body interaction problem, Applied ocean research, 13(6), 317-319, 1991. https://doi.org/10.1016/S0141-1187(05)80055-0

[8] Bai W., Non-linear wave interaction with arbitrary 3-D bodies. PhD thesis, Dalian university of technology, China, 2001.

[9] Teng B., Second-order wave action on 3D floating body. Journal of Hydrodynamics A, 10(3):316-327, 1995.

[10] Song M.J., Kim K.H. and Kim Y.H., Numerical analysis and validation of weakly nonlinear ship motions and structural loads on a modern containership, Ocean engineering, 38, 77-87, 2011. https://doi.org/10.1016/j.oceaneng.2010.09.017 
[11] Shao Y.L, Faltinsen O.M., Linear seakeeping and added resistance analysis by means of body-fixed coordinate system, Journal of marine science and technology, 17(4), 493-510, 2012. https://doi.org/10.1007/s00773-012-0185-y

[12] Zhao R. and Faltinsen O.M., A discussion of the m-terms in the wave-current-body interaction problem, 4th international workshop on water waves and floating bodies, Oystese, Norway, 1989.

[13] Raven H.C., A solution method for the nonlinear ship wave resistance problem. PhD thesis, Delft University of technology, Delft, Netherlands. 1996.

[14] Bingham H.B. and Maniar H.D., Computing the double-body m-terms using a B-spline based panel method, 11th international workshop on waver waves and floating bodies, Hamburg, Germany, 1996.

[15] Duan W.Y., Chen J.K. and Zhao B.B., Second-order Taylor expansion boundary element method for the second-order wave radiation problem, Applied ocean research, 52, 12-26, 2015. https://doi.org/10.1016/j.apor.2015.04.011

[16] W.Y. Duan, F.T. Meng and J.K. Chen. PTEBEM for wave drift forces based on hydrodynamic pressure integration. Engineering analysis with boundary elements, 2020 (Accepted). https://doi.org/10.1016/j.enganabound.2020.10.018

[17] Yasukawa H., Hirata N., Matsumot A., Kuroiwa R. and Mizokami S., Evaluations of wave-induced steady forces and turning motion of a full hull ship in waves. Journal of marine science and technology, 2019, 24:1-15. https://doi.org/10.1007/s00773-018-0537-3

[18] W.Y. Duan, J.D. Li, J.K. Chen and S. Ma. Time-domain TEBEM method for wave added resistance of ships with forward speed. Journal of marine science and technology, 2020, (Published online). https://doi.org/10.1007/s00773-020-00729-2

Submitted:

Accepted:
24.7.2020.

17.12.2020.
J.K. Chen

W.Y. Duan*

duanwenyangheu@sina.com

College of Shipbuilding Engineering, Harbin Engineering University, Harbin, Heilongjiang 150001, China. 